\title{
Scrutinizing the Clinical Symptoms and treatment Outcomes in a Case of Parasomnia with Sleepwalking Disorder Associated with Nocturnal Violence
}

\author{
Selma Pasalari ${ }^{1}$, Kazem Khorramdel ${ }^{1 *}$, Babak Kateb², KS Rao ${ }^{3}$, Mohammad Nami*3,4 \\ ${ }^{1}$ Department of Psychology and Education Science, Fatemiyeh Shiraz, Institute of Higher Education, Shiraz, Iran \\ 2Director, National Center for NanoBioElectoronics; Director, Brain Technology and Innovation Park; President, \\ Brain Mapping Foundation; Chairman and CEO, Society for brain mapping and Therapeutics and, Chairman of \\ Neuroscience20-G20 summit, Los Angeles, CA, USA \\ ${ }^{3}$ Neuroscience Center, Instituto de Investigaciones Científicas y Servicios de Alta Tecnología (INDICASAT AIP), City of \\ Knowledge 084301103, Panama \\ ${ }^{4}$ Department of Neuroscience, School of Advanced Medical Sciences and Technologies, Shiraz University of Medical \\ Sciences, Shiraz, Iran
}

Corresponding author: Kazem Khorramdel PhD, Department of Psychology and Education Science, Fatemiyeh Shiraz, Institute of Higher Education, Shiraz, Iran,

Email: khoramdel.psy@gmail.com; M. Nami MD, PhD, 3Department of Neuroscience, School of Advanced Medical Sciences and Technologies, Shiraz University of Medical Sciences, Shiraz, Iran Email: mtneurosci2@gmail.com

\begin{abstract}
The aim of this study was to investigate a case of sleepwalking associated with violence (non-REM parasomnias) and obstructive sleep apnea-hypopnea syndrome (OSAHS) following treatment strategies. Here we studied a 60-year-old man with family history of a wide range of sleep disorders. His quality of sleep, anxiety, depression, quality of life, and possibility of post-traumatic stress disorder (PTSD) were examined using the standard questionnaires upon pre-treatment, post-treatment, and follow-up phases of the study. The treatment plan comprised adherence to sleep hygiene measures, applying continuous positive airway pressure machine (CPAP) concurrently with eight sessions of weekly biofeedback therapy sessions. Standard over-night polysomnographic evaluations were done prior to and after the treatment. The present report comparatively highlights the patient's sleep bioparameters, number of arousals, respiratory events, and periodic limb movements (PLM) during sleep stages in pre- and post-treatment studies. Prior to the intervention, the subject suffered from OSA, anxiety, minor depression, moderate quality of life and some degree of PTSD resulting in frequent episodes of sleepwalking associated with violence. After the intervention, there was a relative improvement in all indices. The apnea/hypopnea index (AHI) was 33.37 at the beginning of the intervention and decreased to 2.24 after 3 weeks of compliant CPAP therapy. The treatment protocol in this study resulted in complete improvement in some parameters such as PLM and OSAHS and relative improvement in others such as arousal instability and parasomnias including sleep walking associated with violance. The present study puts forward further insights into the possible relation between parasomnias and sleep disordered breathing with intermittent hypoxia. The above hypothesis deserves further investigations in future controlled studies.
\end{abstract}

Keywords: NREM parasomnias, Sleepwalking, Obstructive sleep apnea, Biofeedback therapy, CPAP, Polysomnography 


\section{Introduction}

Sleep is one of the most important parts of human life. Sleep related disorders can potentially result in detrimental health consequences when left untreated. Given the competitive life style in today's modern era, the incidence of sleep-wake disorders is found to be continuously on steep rise to the extent that some reports indicate $64 \%$ of American adults suffer from sleep disorders (1). Other population-based studies have also demonstrated an increased rate of sleep related predicaments over the past two decades (2).

Sleep disorders can lead to excessive daily sleepiness, fatigue, respiratory and other physical problems.

According to the Diagnostic and Statistical Manual of Mental Disorders, fifth Edition (DSM-5), sleep disorders are wide varied in terms of clinical presentation, yet the most prevalent ones include insomnia, hypersomnia, narcolepsy, respiratory sleep disorders, circadian rhythm sleep disorders, nightmare disorder, sleep panic disorder, parasomnia and sleepwalking disorders $(3,4)$.

Non-rapid eye movement (NREM) parasomnias constitute a category of sleep disorders characterized by abnormal behaviors and physiological events primarily observes in stages 2 (N2) and 3 (N3) of NREM sleep (4-6), thus, most often takes place in the first third of the night when these sleep stages predominate, and the individual is deemed outside of his/her conscious awareness (7). Due to their specific association with slow wave sleep (SWS) (N3), NREM parasomnias are also termed 'SWS disorders' (8). Behaviors such as confusional arousals, sleepwalking (somnambulism), night terrors, and sleep-related violence are among the key types of NREM parasomnias which arise during N3. Confusional arousals, sleepwalking and night terrors are also termed 'disorders of arousal', as they share features of autonomic and motor excitability (9).

The potential adverse health consequences of sleepwalking are injury to the sleepwalkers themselves or to others owing to impaired perception and the characteristic of sleepwalking (8).

Sleepwalking associated with violence has been reported to cause significant injury requiring hospitalization or even death. Such an issue is perhaps not sufficiently attended to, potentially due to a low prevalence rate of sleepwalking or in its subsequent adverse events. The condition hence subjects to inadequate identification, reporting, or assessment as the cause of injuries $(8,10)$. Understanding the epidemiology of sleepwalking and its comorbid sleep related problems are deemed to be salient to public health, individual decision making and clinical management.

Somnambulism can consist of very complex motor activity of which walking is just one element. Somnambulism may be precipitated by a variety of conditions such as insufficient sleep resulting from an irregular sleep schedule, staying up late, giving up a daily nap or waking early in the morning (7). Other implicated factors might be fever, stress, medications (phenothiazines, chloral hydrate, lithium) and other disorders which result in arousal instability including the obstructive sleep apnea-hypopnea syndrome (OSAHS) and periodic limb movement during sleep (PLMD) and sleep epilepsies (11, 12).

Moreover, some recent studies report a solid link between psychopathology and somnambulism. Additionally, some reports have also substantiated correlations between NREM parasomnias and mood disorders and anxiety (13). According to selfreported studies almost $25 \%$ of adult sleepwalkers suffer from depressive or bipolar mood disorders (14-16). In populations bearing mental health problems, adult patients with NREM parasomnias experience a higher frequency of psychological episodes with 
diverse and complex clinical features as compared to pediatric patients (17). These clinical findings are important to physicians and health care providers mainly nursing staff and may have implications for the concurrent management strategies both for sleep disorders and associated mental disorders.

Research focusing on NREM parasomnias has started to gain momentum over the past two decades. In recent years, sleep researchers have started to take a more indepth look into this area bringing in further insights into the characteristics, mechanisms, underpinning causes and treatment of NREM parasomnias. Yet, the current state of knowledge about this sleep disorder is still far from complete.

Therefore, the aim of the present study was to comprehensively investigate a 60 yearold male patient who was initially diagnosed with NREM parasomnia accompanied by violence with documented OSAHS upon polysomnographic (PSG) assessment. Furthermore, a treatment plan based on psychological, physical, and pharmacological characteristics was implemented with its effectiveness carefully evaluated over time.

\section{Case presentation}

In the present study, we comprehensively evaluated a 60 year-old war-veteran male patient with a family history of wide range of sleep disorders. He has been reporting wax and wanes in sleep walking, groaning and shouting during sleep since his childhood. After the age of 50, while the frequency of sleepwalking episodes started to steadily decrease, his symptoms turned to be significantly associated with harsh violence upon sleep. He reported difficulty in falling and staying asleep and had either long diurnal naps or excessive daytime sleepiness for the past several years. During times of chronic stress, sleep disorders and violent reactions were reported to be significantly intensified often resulting in self injury or threatening others while asleep. His sexual desire and appetite were normal. The patient was fully debriefed about the diagnosis and treatment processes after which he signed an inform consent to partake in this investigation.

\section{Assessment of psychological status}

Indices including the sleep quality, sleep disordered breathing, anxiety, depression, quality of life and possibility of post-traumatic stress disorder (PTSD) were assessed upon pre-intervention, post-intervention and follow-up time courses. To this end, validated Persian versions of the questionnaires including Berlin Questionnaire, Overall Anxiety Severity and Impairment Scale (OASIS), Beck Depression Inventory-II, Quality of Life Questionnaire of World Health Organization, and the PTSD Assessment Questionnaire were used, respectively.

\section{Treatment plan}

The patient's baseline and follow-up PSG report were interpreted by a sleep disorders fellow. Based on his baseline assessment, the following treatment plan was suggested:

1) The patient was instructed to the principles of sleep hygiene before going to bed as advised by a sleep psychologist.

2) The continuous positive airway pressure (CPAP, BMC Luna Auto CPAP System) was used to rectify sleep disordered breathing ( baseline apnea-hypopnea index of 33.37).

3) Eight sessions of weekly biofeedback therapy sessions were performed using the wireless Nexus 10 device. Biofeedback is a psychophysiological technique that involves the use of visual and auditory feedback to gain control over the body's involuntary functions. Heart rate, muscle strength, blood pulse volume, respiration 
and blood pressure sensors were used, and real-time data were being acquired based on which feedback training was given as per the patient's physical reaction. During the session, the therapist guided the person through various mental exercises comprising visualization, meditation, mindful breathing and relaxation techniques.

His concurrent medicinal therapy prescribed for eight weeks comprised Biperiden $2 \mathrm{mg}$ qhs and Aripiprazole $2.5 \mathrm{mg}$ qhs.

Prior to, and after the conclusion of the therapeutic plan, full night videopolysomnographic sleep study and split-night PAP titrations were performed at an accredited sleep disorders laboratory between 09:30 pm and 06:30 am the next morning. A technician was present during the entire recording. The following data were documented to a special sleep recording computer (NEURON-SPECTRUMAM/PSG) and scored according to the latest AASM manual for sleep scoring and analysis: EEG (19-ch sleep-EEG was employed to rule out any possible epileptiform discharges, the montage was unipolar as per the 10-20 international system for the placement of EEG electrodes), EOG (right), EOG (left), EMG(mouth/nose air flow), snoring sounds, oxygen saturation, plethysmogram, ECG, heart rate and sleeping position.

Recording and scoring of sleep bioparameters and the related statistical analyses generated data on sleep latency, sleep stages, number of arousals, wakes after sleep onset (WASOs), respiratory events in relation to arousals and body position, heart rate and rhythms, blood oxygen saturation, snoring, periodic limb movements during sleep, and sleep bruxism.

\section{Results}

\section{psychological Indicators}

The studied subject suffered from a severe OSAHS and scored positive in all three categories in the Berlin questionnaire prior to the treatments. Meanwhile, at the end of the both at the end of the treatment course and upon 2-month follow-up his Berlin questionnaire turned acquire negative scores in all three categories.

Upon baseline assessments, his overall anxiety severity and mood impairment scales were confirmed at the cut-off point of eight, while after the treatment same scores reduced by $50 \%$ and remained consistent after a two-month follow-up. As such, it appeared that time had no negative effect on the effectiveness of the intervention given at least in our two-month follow-up plan (Figure 1, A).

Before the intervention, the studied subject obtained the score 7 from Beck depression inventory-II, indicating a slight depression, however, after therapeutic interventions and especially in the follow-up time-point, depression score dropped significantly and was close to zero (Figure 1, B).

The quality of life was assessed in four domains including physical health, psychological health, social relationships, and the environment. Our results showed that the intervention improved the physical and psychological health indices, while social relationships and environment did not change after the intervention and upon the follow-up time-point. These results were probably, and at least partly, due to the lack of change in the social relationships and living environment of the studied person given the pandemic situation (Figure 1, C). 
Regarding the PTSD assessment questionnaire, the subject received a relatively high score of 61 before the intervention. Though the score was not at or above the cut-off point for this questionnaire, it was still considered as high. Like other indices, the treatment was able to reduce the score of this test to 31 (Figure 1, D).
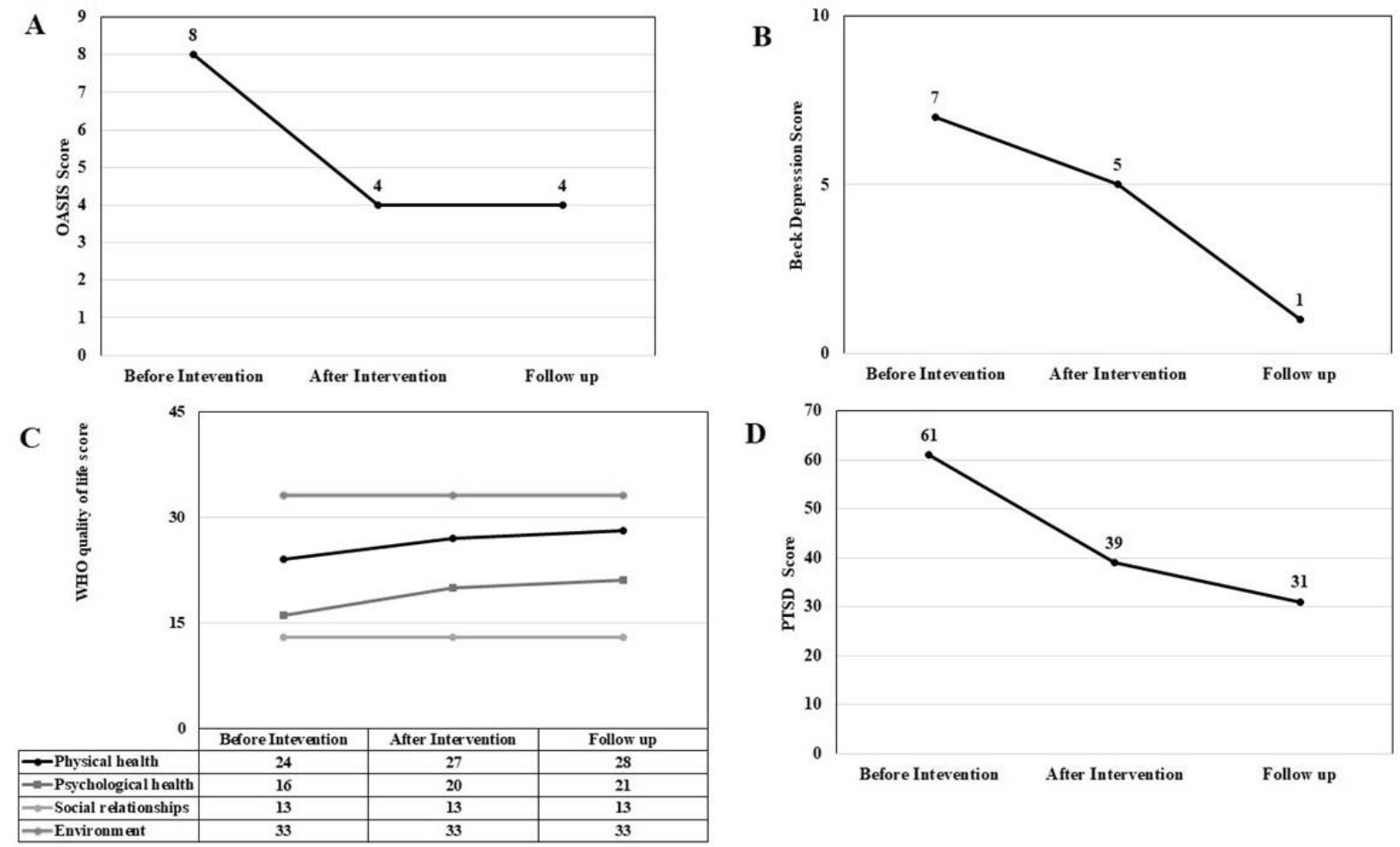

Figure 1: Sleep quality and obstructive sleep apnea, anxiety, depression, quality of life and posttraumatic stress disorder (PTSD) were assessed in the pre-intervention, post-intervention and follow-up stages using Berlin Questionnaire, Overall Anxiety Severity and Impairment Scale (OASIS), Beck Depression Inventory-II, Quality of Life Questionnaire of World Health Organization, and the PTSD Assessment Questionnaire.

\section{Outcomes of the therapeutic interventions}

The CPAP device was used for 36 days. Accordingly, the apnea-hypopnea index (AHI) of 33.37 at the beginning of the CPAP intervention was significantly decreased down to 2.24 on the last day. As the therapeutic process continued, the patient's condition improved and the frequency of respiratory event markedly decreased (Figure 2).

Eight sessions of biofeedback therapy were planned and conducted. To do so, following the necessary preparations, various sensors such as blood volume pulse sensor (BVP), respiratory sensor (RSP) and respiratory rate sensor (RSP-Pacer) were connected to the patient and various feedback and biological signals were recorded. Finally, in the output curves of the device, the remediative effects of eight sessions of biofeedback therapy on cardiovascular and respiratory indices were observed, which remained consistent with the post-intervention polysomnography report of the patient. 

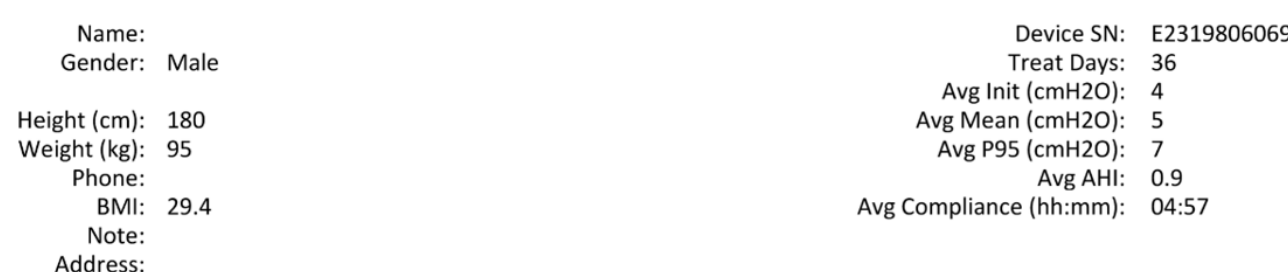

Pressure (cmH2O) $122 \quad 3 \quad 4 \quad 5 \quad 6 \quad 788910111213141516171819202122232425$

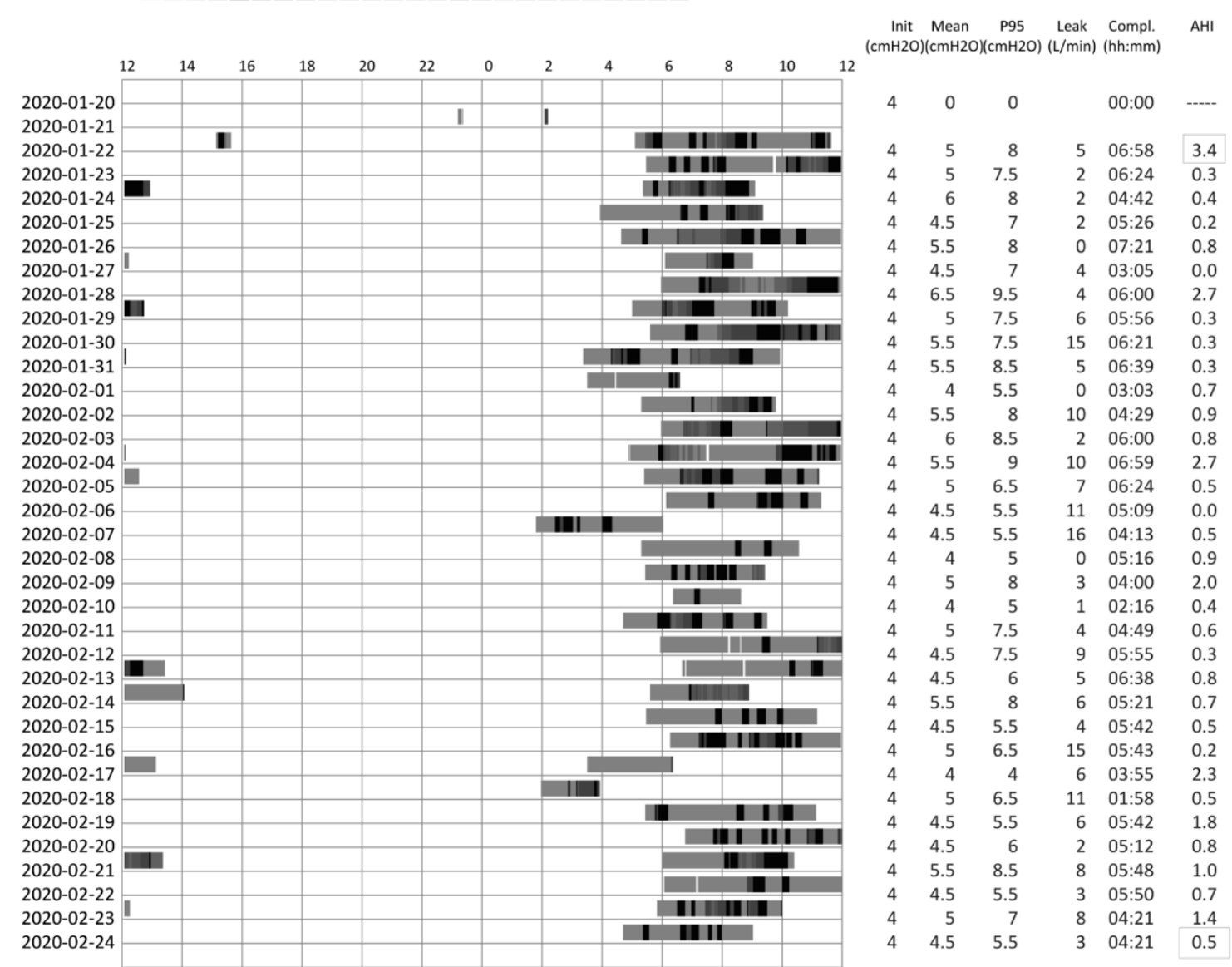

Figure 2. Apnea/hypopnea index (AHI)-Chart upon baseline and following compliant CPAP use/follow-up

Having studied the PSG indices before therapeutic interventions, there were frequent wakes after sleep onset (WASOs) was 28, among which 16 awakenings lasted for over $3 \mathrm{~min}$. The total time in bed (TIB) was $7 \mathrm{~h} 53$ min while the total sleep time (TST) was $3 \mathrm{~h} 13 \mathrm{~min}$. Therefore, the sleep efficiency index that obtained from the division of TST to TB, showed a rate of $40 \%$, which represent very poor sleep efficiency. The total number of arousals was also reported 434 times and the arousal index obtained by dividing the total number of arousals by the total number of hours of sleep was recorded as 33.71 (Normal<5) (Table 1).

Following the treatment, number of WASOs was reduced to 9 times. In addition, WASOs lasting more that 3-min were reduced to 11 times. After intervention, TIB was $8 \mathrm{~h} 16 \mathrm{~min}$ and TST was 5:21'. Accordingly, SE reached to $64 \%$. The total number of arousals decreased to 66 times and as such, the arousal index showed a significant decrease down to 6.94 (Table 1). 
Scoring the patient's bruxism episodes before treatment revealed 543.1 bruxism per hour (2299 episodes of bruxism in total), which collectively lasted for 10':21" during NREM sleep. After therapeutic interventions, the number of bruxism per hour was significantly reduced to only 9.1. The total number of episodes was 49 timesthey collectively lasted for only 8 seconds (Table 2).

Table 1. Sleep statistics of studied subject before and after therapeutic interventions

\begin{tabular}{lll}
\hline Parameters & Before Interventions & After Interventions \\
\hline Time in bed (TIB) & $12: 53: 11$ & $11: 36: 40$ \\
Total sleep period time (TSPT) & $12: 52: 30$ & $09: 31: 00$ \\
Start of sleep period & $9: 43: 21 \mathrm{AM}$ & $11: 54: 30 \mathrm{AM}$ \\
End of sleep period & $10: 35: 51 \mathrm{PM}$ & $9: 52: 30$ PM \\
Total sleep time (TST) & $04: 13: 30$ & $05: 21: 30$ \\
Awake time after sleep onset & $06: 27: 00$ & $04: 43: 30$ \\
Total wake time during sleep period & $06: 27: 00$ & $04: 09: 30$ \\
Number of awakenings & 28 & 21 \\
Number of awakenings with more than 3 minute & 16 & 11 \\
duration & $32.8 \%$ & $46.1 \%$ \\
Sleep efficiency index (TST/TB) & 434 & 66 \\
Number of arousals & 33.71 & 6.94 \\
Arousal Index & & \\
\hline
\end{tabular}

Table 2. Bruxism analysis of the studied subject before and after therapeutic interventions

\begin{tabular}{|c|c|c|c|c|c|c|}
\hline \multirow{2}{*}{ Parameters } & \multicolumn{2}{|l|}{ NREM } & \multicolumn{2}{|l|}{ REM } & \multicolumn{2}{|c|}{ NREM+REM } \\
\hline & Before & After & Before & After & Before & After \\
\hline $\begin{array}{l}\text { Total duration of } \\
\text { bruxism episodes }\end{array}$ & $00: 10: 21$ & 00:00:08 & 00:00:00 & 00:00:00 & 00:10:21 & 00:00:08 \\
\hline $\begin{array}{l}\text { Total number of } \\
\text { bruxism episodes } \\
\text { Number of } \\
\text { bruxism per hour } \\
\text { (index) }\end{array}$ & $\begin{array}{l}2299 \\
543.1\end{array}$ & $\begin{array}{l}48 \\
9.1\end{array}$ & 0 & 1 & $\begin{array}{l}2299 \\
543.1\end{array}$ & $\begin{array}{l}49 \\
9.1\end{array}$ \\
\hline
\end{tabular}

The analysis of snores upon different stages of sleep before the treatment documented 3334 snores during REM and NREM sleep, regardless of the sleeping position. Notably, this was decreased to 234 following the treatment (Table 3).

In addition to the above, the analysis of respiratory events, as well as the number of arousals related to such events are summarized in Table 4. Prior to therapeutic interventions, the number of obstructive apneas and hypopneas were 34 (4.5\% of total sleep time) and 106 (16.65\% of total sleep time), respectively. Meanwhile, the treatment eliminated all apneas and obstructive hypopnea events were limited to only 12 times (1.13\% of the total sleep time) (Figure 3 ).

Concerning the periodic limb movements during different stages of sleep (PLMD), the baseline PLM index of 5.04 (Figure 4, A) was eliminated after the treatment and the patient was found to have unwanted limb movements during sleep (Figure 4, B). 
Table 3. Snore analysis of the studied subject before and after therapeutic interventions

\begin{tabular}{|c|c|c|c|c|c|c|}
\hline \multirow{2}{*}{ Parameters } & \multicolumn{2}{|l|}{ NREM } & \multicolumn{2}{|l|}{ REM } & \multicolumn{2}{|c|}{ NREM+REM } \\
\hline & Before & After & Before & After & Before & After \\
\hline $\begin{array}{l}\text { Total snore } \\
\text { duration }\end{array}$ & 00:03:46 & 00:02:06 & 00:00:00 & 00:00:14 & 00:03:46 & $00: 02: 20$ \\
\hline $\begin{array}{l}\text { Number of } \\
\text { snores }\end{array}$ & 334 & 215 & 0 & 19 & 334 & 234 \\
\hline $\begin{array}{l}\text { Number of } \\
\text { snores per hour } \\
\text { (index) }\end{array}$ & 78.9 & 40.9 & - & 190 & 78.9 & 43.7 \\
\hline
\end{tabular}

Table 4. Respiratory events of the studied subject before and after therapeutic interventions

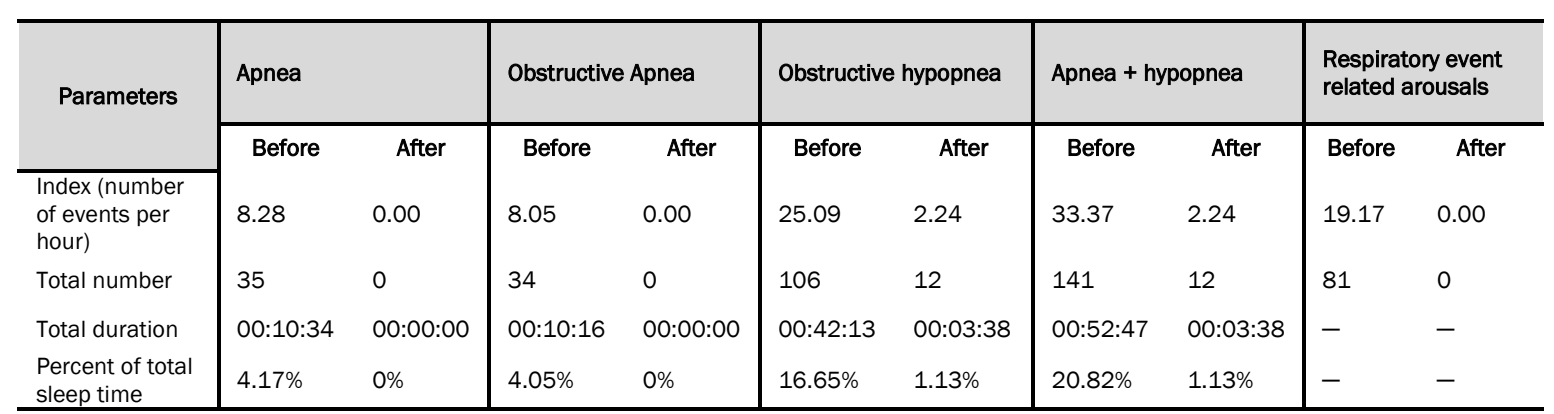

\section{Respiratory event index}

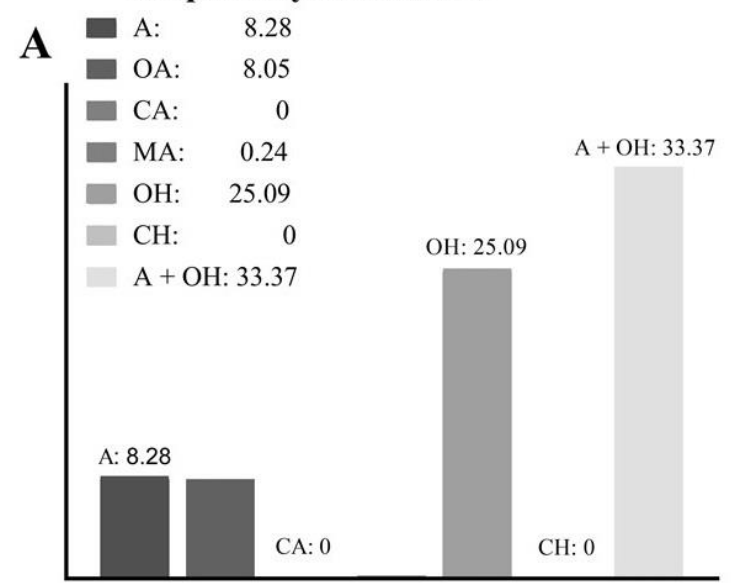

\section{Respiratory event index}

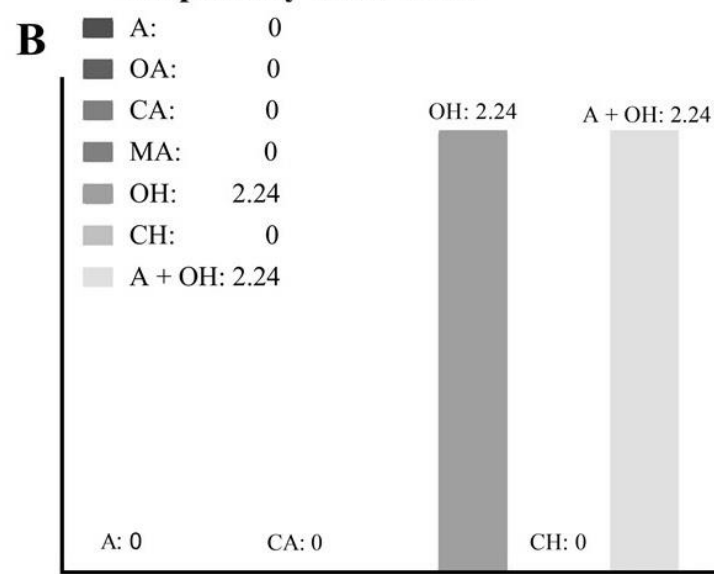

Figure 3: Respiratory event index (events per hour). (A) Before therapeutic interventions and (B) After therapeutic interventions. A: apnea, OA: obstructive apnea, CA: central apnea, MA: mixed apnea, $\mathrm{OH}$ : obstructive hypopnea, $\mathrm{CH}$ : central hypopnea, $\mathrm{A}+\mathrm{OH}$ : apneas + obstructive hypopneas. 

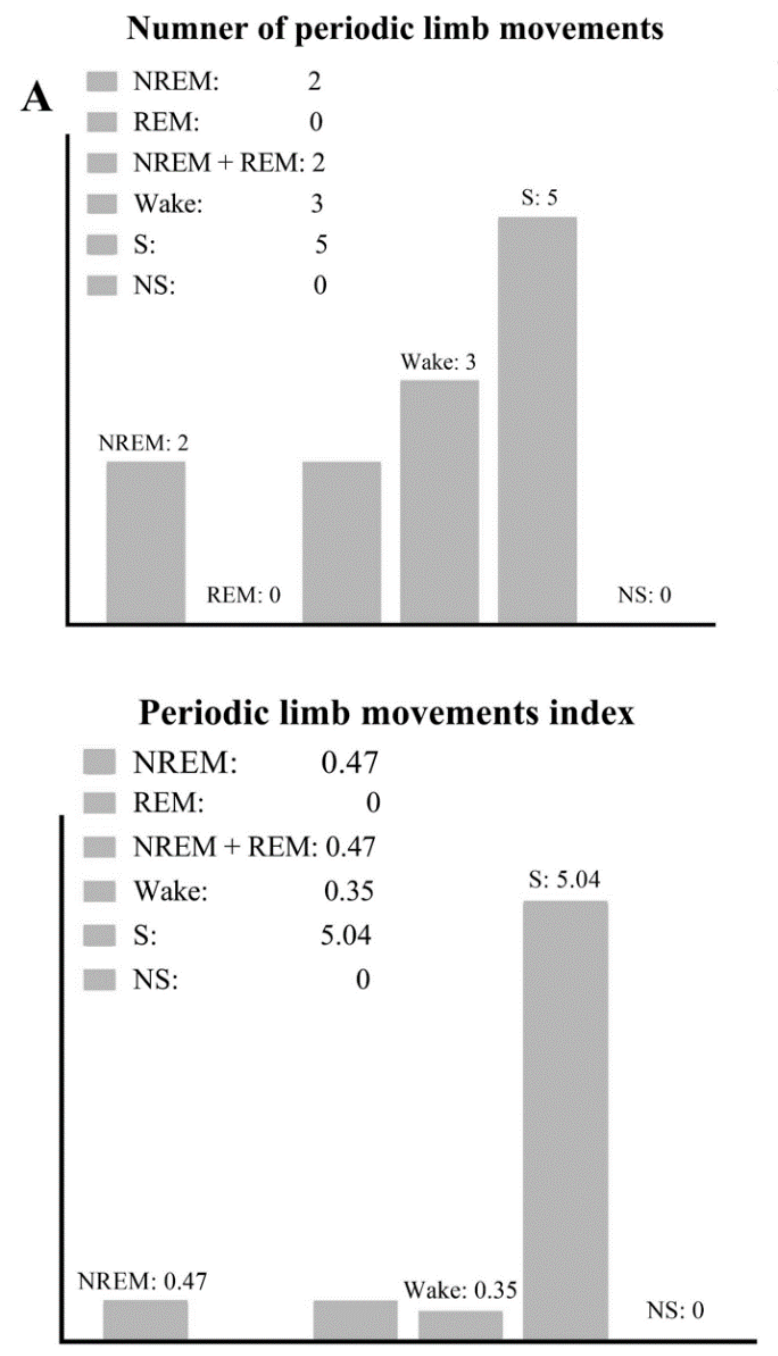

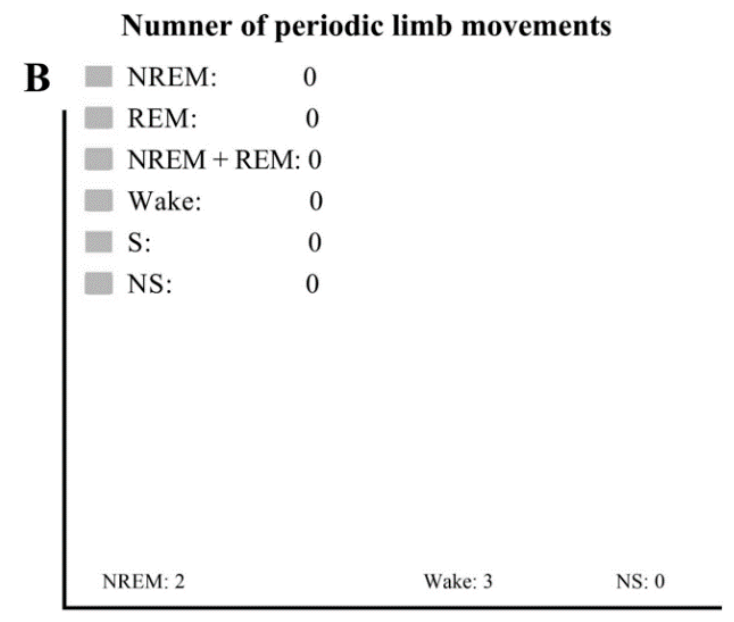

\section{Periodic limb movements index}

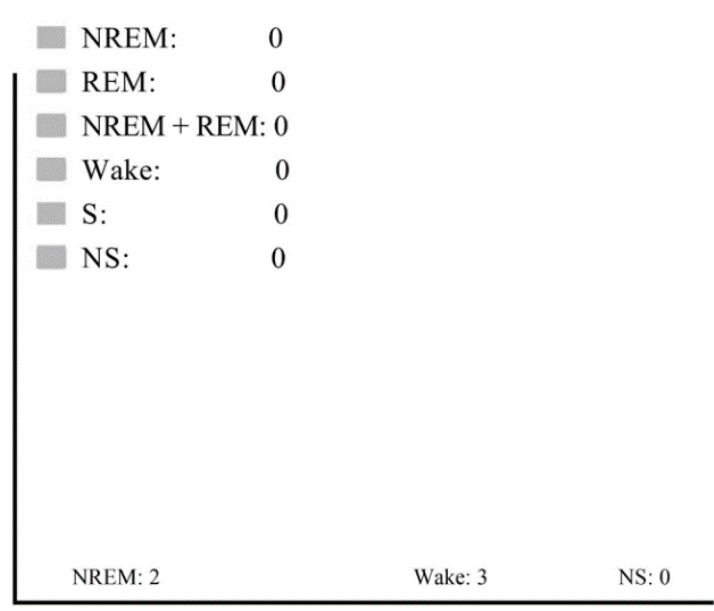

Figure 4: Periodic limb movements frequency and index, (A) Before therapeutic interventions and (B) After therapeutic interventions.

\section{Discussion}

The present case investigation proposed that an appropriately planned combination therapy intervention including sleep hygiene, limited medicinal therapy, and use of CPAP and biofeedback devices where indicated retains the potential to significantly improve behavioral sleep disorders including NREM parasomnias associated with violence. The present case could have seen almost complete elimination of sleep disordered breathing upon an ongoing use of CPAP. A pronounced improvement in behavioral sleep symptoms could partly be due to less frequent intermittent hypoxia events and regulated sleep bioparameters reflecting in a decreased sympathetic tone.

Unfortunately, while the incidence of sleep related disorders including parasomnias is rising ( specifically since the pandemic began), such conditions subject to an inadequate clinical attention. According to the Center for Disease Control, more than a third of adults in the United States do not get enough sleep and many sleep disorders root in comorbidities such as anxiety or depressive disorders (18). Therefore, recognizing the underlying causes of sleep predicaments and deciding on the proper treatment strategies need to be further attended to by the physicians and nursing staff.

In this regard, a single-armed clinical study 512 patients with NREM parasomnia (19) found that the first line of effective treatment in these patients is sleep hygiene and 
secondly the management of underlying factors such as stress and anxiety and finally pharmacological treatments where indicated. The results of this study showed that in over $30 \%$ on the instances, non-pharmacological therapies are much more useful and effective than pharmacological treatments. The results of the present study also showed that adherence to sleep hygiene and regular sleep-wake schedule along with other interventions in this patient's personalized treatment plan could effectively improve his significant sleep related symptoms.

In another study, CPAP had no effect on reducing periodic limb movements (PLM) in people with OSAHS. However, several indicators of sleep problems were significantly improved upon the nocturnal use of CPAP (20). In contrast, our findings revealed the completely rescinded PLMD symptoms following the compliant use of CPAP. Some studies have also highlighted the association of PLM and arousal instability during sleep $(21,22)$. In line with the above, our case study showed that the use of CPAP in combination with other interventions was able to significantly reduce the frequency and duration of arousals and eliminated PLM both during REM and NREM sleep.

The reason behind using biofeedback in this specific case was to pursue polyvagal therapy which is known to help hypnic tone. Overall, biofeedback therapy is used in psychosomatic, anxiety, and neurological disorders. Biofeedback has been shown to improve autonomic symptoms such as neurotic hypertension and irritable bowel syndrome. The EEG biofeedback therapy (neurofeedback) is also shown to be effective in attention deficit hyperactivity disorder (ADHD) and anxiety-related symptoms. Other studies have shown that the use of psychological approaches such cognitive-behavioral therapies in the treatment of adult sleep parasomnia have beneficial effects (23-25). Based on a recent report, biofeedback therapy is found capable in significantly alleviating the motor activity associated with bruxism during sleep (23). Some studies have also demonstrated the effects of biofeedback use vs. sham in reducing the sleep latency and the number of WASOs in patients with chronic psychophysiological insomnia (26).

In the present study, biofeedback therapy was used in combination with other therapeutic interventions in a case of NREM parasomnia yielding a proper clinical outcome.

Recent pathophysiological findings in sleep structure of cases with parasomnias have helped researchers better understand several mechanisms of drugs' mode of action. For instance, evidence-based medicine supports the fact that benzodiazepines, antidepressants, and L-5-hydroxytryptophan are helpful in disorders of arousals (DOA). On the other hand, selective serotonin reuptake inhibitors (SSRIs) and topiramate are found effective in sleep-related eating disorders (SREDs). REM sleep behavioral disorders (RBDs) respond to clonazepam, melatonin, and to a lesser extent to dopaminergic and anticholinergic medications. Prazosin and cannabinoids are effective in nightmare disorders. Sleep paralysis may also respond to antidepressants and tricyclic antidepressant may be effective in sleep-induced hallucinations (27).

In the present case study, the use of Biperiden and Aripiprazole could have beneficial effects in relieving the patients' symptoms. Meanwhile the proportional effects of each of the modalities in the patient's treatment plan is not examined here and requires sham- or placebo-controlled clinical investigations. 


\section{Conclusions}

Personalized approaches in alleviating symptoms of parasomnia need to be emphasized since patients may present with a wide-varied clinical symptom. The presence of comorbidities (OSAHS and marginal symptoms compatible with PTSD in this case) need to be carefully considered and addressed. More case reports, case series and optimally, controlled clinical trials need to be pursued to suggest evidence-based clinical decision-making trees in treating parasomnia cases with or without comorbidities.

\section{Acknowledgments}

The authors acknowledge A.M. Kamali PhD and M. Kazemiha PhDc at Dana Brain Health Institute for their assistance and support. We also sincerely thank the patient who cooperated with us in all stages of the work and wish him good health and a sustainable recovery.

\section{References}

1. Akerstedt T, Knutsson A, Westerholm P, Theorell T, Alfredsson L, Kecklund G. Sleep disturbances, work stress and work hours: a cross-sectional study. J Psychosom Res. 2002;53(3):741-8.

2. Yudofsky SC, Hales RE. Essentials of Neuropsychiatry and Clinical Neurosciences: American Psychiatric Pub.; 2004.

3. Thorpy MJ. Classification of sleep disorders. Neurotherapeutics. 2012;9(4):687-701.

4. Association AP. Diagnostic and Statistical Manual of Mental Disorders (DSM-5®): American Psychiatric Publishing; 2013.

5. Espa F, Ondze B, Deglise P, Billiard M, Besset A. Sleep architecture, slow wave activity, and sleep spindles in adult patients with sleepwalking and sleep terrors. Clin Neurophysiol. 2000;111(5):929-39.

6. Hrozanova M, Morrison I, Riha RL. Adult NREM Parasomnias: An Update. Clocks Sleep. 2018;1(1):87-104.

7. Kuhn BR, Elliott AJ. Treatment efficacy in behavioral pediatric sleep medicine. J Psychosom Res. 2003;54(6):587-97.

8. Stallman HM, Kohler M. Prevalence of Sleepwalking: A Systematic Review and MetaAnalysis. PLoS One. 2016;11(11):e0164769.

9. Broughton RJ. Sleep disorders: disorders of arousal? Enuresis, somnambulism, and nightmares occur in confusional states of arousal, not in "dreaming sleep". Science. 1968;159(3819):1070-8.

10. Mume CO. Prevalence of sleepwalking in an adult population. Libyan J Med. 2010;5:10.4176/090907.

11. Lavie P, Pillar G, Malhotra A. Sleep Disorders Handbook: A Handbook for Clinicians: Taylor \& Francis; 2002.

12. Bharadwaj R, Kumar S. Somnambulism: Diagnosis and treatment. Indian J Psychiatry. 2007;49(2):123-5.

13. Selby KE, Morrison I, Riha RL. Psychiatric comorbidity in arousal disorders: chicken or egg? J Neuropsychiatry Clin Neurosci. 2012;24(3):E36.

14. Labelle MA, Dang-Vu TT, Petit D, Desautels A, Montplaisir J, Zadra A. Sleep deprivation impairs inhibitory control during wakefulness in adult sleepwalkers. J Sleep Res. 2015;24(6):658-65.

15. Bargiotas P, Arnet I, Frei M, Baumann CR, Schindler K, Bassetti CL. Demographic, Clinical and Polysomnographic Characteristics of Childhood- and Adult-Onset Sleepwalking in Adults. Eur Neurol. 2017;78(5-6):307-11. 
16. Ohayon MM, Guilleminault C, Priest RG. Night terrors, sleepwalking, and confusional arousals in the general population: their frequency and relationship to other sleep and mental disorders. J Clin Psychiatry. 1999;60(4):268-76; quiz 77.

17. Lam SP, Fong SY, Yu MW, Li SX, Wing YK. Sleepwalking in psychiatric patients: comparison of childhood and adult onset. Aust N Z J Psychiatry. 2009;43(5):426-30.

18. Bjorvatn B, Grønli J, Pallesen S. Prevalence of different parasomnias in the general population. Sleep Med. 2010;11(10):1031-4.

19. Drakatos P, Marples L, Muza R, Higgins S, Gildeh N, Macavei R, et al. NREM parasomnias: a treatment approach based upon a retrospective case series of 512 patients. Sleep Med. 2019;53:181-8.

20. Hedli LC, Christos P, Krieger AC. Unmasking of periodic limb movements with the resolution of obstructive sleep apnea during continuous positive airway pressure application. J Clin Neurophysiol. 2012;29(4):339-44.

21. Allena M, Campus C, Morrone E, De Carli F, Garbarino S, Manfredi C, et al. Periodic limb movements both in non-REM and REM sleep: relationships between cerebral and autonomic activities. Clin Neurophysiol. 2009;120(7):1282-90.

22. Walters AS, Lavigne G, Hening W, Picchietti DL, Allen RP, Chokroverty S, et al. The scoring of movements in sleep. J Clin Sleep Med. 2007;3(2):155-67.

23. Jokubauskas L, Baltrušaitytė A. Efficacy of biofeedback therapy on sleep bruxism: $A$ systematic review and meta-analysis. J Oral Rehabil. 2018;45(6):485-95.

24. Markiewcz R. The use of EEG Biofeedback/Neurofeedback in psychiatric rehabilitation. Psychiatr Pol. 2017;51(6):1095-106.

25. Galbiati A, Rinaldi F, Giora E, Ferini-Strambi L, Marelli S. Behavioural and CognitiveBehavioural Treatments of Parasomnias. Behav Neurol. 2015;2015:786928.

26. Melo DLM, Carvalho LBC, Prado LBF, Prado GF. Biofeedback Therapies for Chronic Insomnia: A Systematic Review. Appl Psychophysiol Biofeedback. 2019;44(4):259-69.

27. Manni R, Toscano G, Terzaghi M. Therapeutic Symptomatic Strategies in the Parasomnias. Curr Treat Options Neurol. 2018;20(7):26. 
